targeting immunization of workers born in 1957 or later because they probably do not have natural immunity from prior infection. Nonetheless, screening of healthcare workers born before 1957 for measles immunity still may be warranted because $30 \%$ of healthcare workers with reported measles in 1991 were born before 1957.

FROM: Centers for Disease Control and Prevention. Measles Surveillance-United States, 1991. In: CDC Surveillance Summaries. M M WR 1992;41 (no. SS-6):1-12.

\section{Patients with Community-Acquired Hepatitis C Have High Rates of Chronic Hepatitis}

Chronic hepatitis was found to develop in a high proportion $(62 \%)$ of patients with community-acquired hepatitis $\mathrm{C}$. This research finding was reported by Miriam J. Alter, $\mathrm{PhD}$, et al of the CDC's Sentinel Counties Chronic Non-A, Non-B Study Team ${ }^{1}$ and provides evidence that the rate of chronic hepatitis following community-acquired hepatitis $\mathrm{C}$ is not, as previously believed, significantly lower than the rate following posttransfusion hepatitis $\mathrm{C}$.

Further, severe disease in the form of chronic active hepatitis was more likely to be found in patients who acquired their infection through blood transfusion, suggesting that the size of the infectious inoculum may be associated with the severity of chronic liver disease.

Non-A, non-B hepatitis accounts for approximately $25 \%$ of acute viral hepatitis in the United States, and $\mathrm{HCV}$ appears to be the etiologic agent of at least $82 \%$ of these cases. Parenteral sources of transmission (ie, injection-drug use, transfusion, and occupational exposure) are risk factors for both non-A, non-B hepatitis and HCV infection. Since 1985, however, transfusions account for only $4 \%$ of the cases of hepatitis C. Other risk factors identified with $\mathrm{HCV}$ infection include household or sexual contact with an infected person and multiple sexual partners. In addition, low socioeconomic level is associated with a large proportion (an average of 30\%) of cases of both non-A, non-B hepatitis and hepatitis $\mathrm{C}$. This risk factor is probably a surrogate marker for other routes of transmission and makes prevention of disease difficult because of its nonspecific nature.
The uniformly high rate of chronic hepatitis found in patients after HCV infection suggests that HCV may be a major cause of chronic liver disease in the United States. In most patients, HCV infection seems to persist for at least several years, even in the absence of active liver disease, emphasizing the urgent need for development of preventive strategies for those in risk groups unassociated with blood transfusions.

\section{RE F E R E N CE}

1. Alter MJ, Margolis HS, Krawczynski K, et. al. The natural history of community-acquired hepatitis $\mathrm{C}$ in the United States. N Engl J

\section{New Procedure Allows Detection of HIV in Blood of Nearly All Infected Persons}

A new procedure developed by researchers at the University of California, San Francisco (UCSF), appears optimal for detecting HIV in the blood on infected individuals. In the past, free human immunodeficiency virus (HIV) could be found in the plasma of only about $35 \%$ of infected people, reported Dr. Li-Zhen Pan et al at UCSE Effective detection of of the virus depends on using an enzyme-linked immunosorbent assay (ELISA) for p24 core antigen of HIV and adding the HIV-infected plasma to target cells.

This testing procedure has confirmed that free virus can be detected in the plasma of almost all individuals infected with HIV if the blood is analyzed within three hours after it is drawn. The researchers confirmed the previous work of other researchers that levels of virus reflect patients' clinical status, with healthier people having less virus and those with very high CD4 + cell counts having less but still detectable virus.

The three-hour window is important for accurate results because beyond this time period substances in the blood such as neutralizing antibodies begin to inactivate the virus so that it is undetectable. However, if plasma and serum samples are frozen and stored at $-70^{\circ} \mathrm{C}$, the virus will remain detectable for up to several months. The researchers noted that this procedure is not suitable for initial HIV screening because it is a lengthy procedure. FROM: Pan L, Werner A, Levy JA. Detection of plasma viremia in human immunodeficiency virus-infected individuals at all clinical stages. J Clin Microbiol.1993;31:283-288. 